\title{
Something old, something new, something borrowed...
}

\author{
Louis Valiquette MD MSc FRCPC 1 , Kevin B Laupland MD MSc FRCPC ${ }^{2,3}$
}

A lthough knowledge of the complex interactions within our intestinal microbiota is relatively new, fecal microbiota transplantation (FMT) has been practiced for a long time. For more than a millennium, human fecal suspensions have been used to treat various ailments. The first literary evidence of oral fecal administration for the treatment of food poisoning and diarrhea dates back to the fourth century in China as detailed in the first Chinese handbook of emergency medicine written by Ge Hong (1). Later, in the 16th century, Li Shizhen described the use of several different human stool preparations for the treatment of various gastrointestinal diseases (1). The first report of FMT for the treatment of pseudomembranous colitis dates back to 1958 (2). In this case series involving four patients, all responded spectacularly. In 1983, Schwan et al (3) published the first report of FMT in a patient with a confirmed Clostridium difficile infection (CDI). Since then, the experiences of more than $250 \mathrm{CDI}$ patients treated with FMT has been published in the medical literature, with an overall success rate of approximately 90\% (4,5).

A hypervirulent strain of $\mathrm{C}$ difficile (NAP1/BI/027) has plagued several hospitals across Canada since 2003. This strain is associated with disease of increased severity, and higher rates of mortality and recurrence compared with other strains $(6,7)$. In general, most patients with CDI will respond to antimicrobial therapy with oral metronidazole or vancomycin. However, recurrence is common and represents one of the most challenging situations in treating CDI patients. Furthermore, some patients will present with a succession of recurrences, which has a major impact on both their quality of life and utilization of health care resources. For example, from 1998 to 2008 in Sherbrooke, Quebec, more than 1300 CDI episodes occurred and, of these, one-third experienced one recurrence, one-third of these experienced a second recurrence, and one-third a third episode and so on (unpublished data).

To treat patients with recurrent disease, several different therapeutic approaches have been suggested; however, none are supported by empirical data. Many experts recommend the use of a tapering or pulsed regimen of oral vancomycin (8). Recently, a new antibiotic, fidaxomicin, has been shown by two multicentric randomized clinical trials to significantly decrease the risk of a first recurrence $(9,10)$. When compared with patients receiving a standard regimen of oral vancomycin, patients treated with fidaxomicin experienced an absolute recurrence risk reduction of $9.8 \%$ in the first trial and $14.2 \%$ in the second trial (both in modified intention to treat analysis). In a subgroup analysis, patients who experienced a single episode of recurrence within three months of enrollment demonstrated similar results (11). Apart from some anecdotal cases, data are lacking to support fidaxomicin use in patients who experience multiple recurrences (12). The unproven efficacy in patients infected with the NAP1 strain is also a limitation in some of these individuals.

Until recently, FMT was only supported by case series and by systematic reviews of case series, with all of the limitations inherent in these types of studies. However, in February 2013, stimulating data were published by van Nood et al (13). The investigators completed an open-label randomized clinical trial in which they initially planned to enroll 120 patients. The main outcome was the absence of relapse at 10 weeks. The three arms of the study compared 13 patients with high-dose vancomycin (500 mg orally for 14 days), 13 patients with high-dose vancomycin (for 14 days) and bowel lavage, and 16 patients with an abbreviated high-dose vancomycin regimen (four to five days) and bowel lavage with FMT administered via a nasogastric tube. The trial was stopped by the data and safety monitoring board on the basis of superior FMT outcome after 43 patients (one was excluded) had been enrolled over a 28 -month period. In fact, 13 FMT patients ( $81 \%)$ were cured on their first infusion compared with seven $(27 \%)$ in the combined control arms. Three patients, who did not respond initially to their first infusion, received a second administration and two of them were cured, yielding a combined efficacy of $94 \%$ in favour of FMT.

There are a number of other important findings and considerations arising from this trial. First, the investigators compared the fecal flora between donors and enrollees (before and after FMT). The flora diversity scores were highly similar between donors and enrollees after FMT, indicating the FMT had recreated a healthy, diverse flora. Second, they offered off-protocol FMT to patients who were initially enrolled in the non-FMT arms and who relapsed. Fifteen of 18 patients were cured using the off-protocol FMT ( $83 \%$ success rate). In spite of these landmark results, some limitations must be addressed. Even if the authors referred to the vancomycin regimen as 'standard', it is clearly not the general approach suggested in published guidelines (8). High-dose vancomycin has never been shown to be superior in the treatment of relapsing CDI and could have had a negative impact on the fecal flora compared with a lower dose. Furthermore, some authors have suggested that high colonic concentrations of vancomycin could be inhibitory to Bacteroides and Bifidobacteria species (14). It is also important to recognize that the proportion of patients infected with ribotype 027 strains was very low in this clinical trial. Consequently, these results might have been different in Canada or other jurisdictions where the prevalence rate of ribotype 027 infection remains high. A group of investigators from Toronto (Ontario) is currently conducting a trial to compare vancomycin tapering with standard vancomycin plus FMT via enema (clinicaltrials.gov).

Although FMT was occasionally practiced before publication of the trial by van Nood et al (13), since this paper was released, several centres across Canada have started or are in the process of developing FMT clinics. However, several questions and challenges remain. Depending of the technique used, FMT can be relatively invasive (eg, total colonoscopy or insertion of a nasogastric tube) and may necessitate specific expertise and/or setting. Moreover, a donor needs to be identified and screened for transmissible diseases. To facilitate the process, donors are usually family members. Recent data from FMT using frozen stools of an unrelated screened donor has shown to be as effective as FMT using fresh stools from a family member (15). Screening varies according to published protocols, but usually involves stool testing (ova and parasites, bacterial cultures, and $\mathrm{C}$ difficile toxin $\mathrm{A}$ and $\mathrm{B}$ testing) and serum testing (HIV-1, HIV-2, hepatitis A, B and $\mathrm{C}$, and rapid plasma reagin). Donors with risk factors for these

${ }^{1}$ Department of Microbiology-Infectious Diseases, Université de Sherbrooke, Sherbrooke, Quebec; ${ }^{2}$ Departments of Medicine, Critical Care

Medicine, Pathology and Laboratory Medicine and Community Health Sciences, University of Calgary, Calgary, Alberta; ${ }^{3}$ Department of

Medicine, Royal Inland Hospital, Kamloops, British Columbia

Correspondence: Dr Louis Valiquette, Université de Sherbrooke, 3001 12ème Avenue Nord, Sherbrooke, Quebec J1H 5N4.

Telephone 819-346-1110 ext 12568,fax 819-829-3289,e-mail louis.valiquette@usherbrooke.ca 
infections should be excluded a priori (intravenous drug user, returned traveller from endemic regions, etc). There are almost as many techniques of stool preparation as practitioners performing FMT (16). We believe that the technique does not need to be overly complicated. It should involve fresh stools mixed with saline or water and be instilled via a fecal enema. For this approach, the key element is volume. A minimum volume of $300 \mathrm{~mL}$ has been suggested by some authors while others have used as much as $1.5 \mathrm{~L}$ of infusion (16). Anecdotally, the results are generally highly favourable regardless of the technique used, even when self-administered at home (17)! The largest challenge lies mostly in convincing patients, potential donors and health professionals to overcome the 'ick' factor associated with FMT.

A group of Ontario investigators recently published information about a stool substitute to be used in FMT known as 'RePOOPulate' (18). This preparation includes 33 different intestinal bacteria isolated in pure culture from a single healthy donor. It has been sucessfully administered to two patients with recurrent CDI. If this approach is found to be efficacious, it could serve as an alternative to fresh stool

\section{REFERENCES}

1. Zhang F, Luo W, Shi Y, Fan Z, Ji G. Should we standardize the 1,700-year-old fecal microbiota transplantation? Am J Gastroenterol 2012;107:1755 (Author reply page 6).

2. Eiseman B, Silen W, Bascom GS, Kauvar AJ. Fecal enema as an adjunct in the treatment of pseudomembranous enterocolitis. Surgery 1958;44:854-9.

3. Schwan A, Sjolin S, Trottestam U, Aronsson B. Relapsing Clostridium difficile enterocolitis cured by rectal infusion of homologous faeces. Lancet 1983;2:845.

4. Gough E, Shaikh H, Manges AR. Systematic review of intestinal microbiota transplantation (fecal bacteriotherapy) for recurrent Clostridium difficile infection. Clin Infect Dis 2011;53:994-1002.

5. Kassam Z, Lee CH, Yuan Y, Hunt RH. Fecal microbiota transplantation for Clostridium difficile infection: Systematic review and meta-analysis. Am J Gastroenterol 2013;108:500-8.

6. Pepin J, Valiquette L, Alary ME, et al. Clostridium difficile-associated diarrhea in a region of Quebec from 1991 to 2003: A changing pattern of disease severity. CMAJ 2004;171:466-72.

7. Loo VG, Poirier L, Miller MA, et al. A predominantly clonal multiinstitutional outbreak of Clostridium difficile-associated diarrhea with high morbidity and mortality. N Engl J Med 2005;353:2442-9.

8. Cohen SH, Gerding DN, Johnson S, et al. Clinical practice guidelines for Clostridium difficile infection in adults: 2010 update by the Society for Healthcare Epidemiology of America (SHEA) and the Infectious Diseases Society of America (IDSA). Infect Control Hosp Epidemiol 2010;31:431-55.

9. Cornely OA, Crook DW, Esposito R, et al. Fidaxomicin versus vancomycin for infection with Clostridium difficile in Europe, Canada, and the USA: A double-blind, non-inferiority, randomised controlled trial. Lancet Infect Dis 2012;12:281-9.

10. Louie TJ, Miller MA, Mullane KM, et al. Fidaxomicin versus vancomycin for Clostridium difficile infection. N Engl J Med 2011;364:422-31 with no risk of disease transmission, a standardized inoculum and with limited repulsive factor. However, normal stool is comprised of more than 4000 different bacterial species, many of which are exceedingly difficult to culture in a laboratory (19). Furthermore, interactions between the normal flora and colonic mucosal cells are complex. Therefore, at this point, it is very difficult predict whether this approach will be successful clinically. It is motivating to know that the same research team is conducting a clinical trial (clinicaltrials.gov).

After more than 1700 years of experience, FMT has only recently been supported by experimental clinical trial evidence in CDI. While proponents of FMT have argued that there may be role for FMT in the treatment of other diseases, such as inflammatory bowel disease, obesity and diabetes mellitus, evidence supporting its use beyond recurrent $\mathrm{CDI}$ is lacking. We now have the clinical trial data to support what we expected from previous anecdotal experience. The challenge is now to refine and optimize FMT protocols and search for comparable standardized culture-based biotherapies for CDI.

11. Cornely OA, Miller MA, Louie TJ, Crook DW, Gorbach SL. Treatment of first recurrence of Clostridium difficile infection: Fidaxomicin versus vancomycin. Clin Infect Dis 2012;55(Suppl 2):S154-61.

12. Johnson S, Gerding DN. Fidaxomicin "chaser" regimen following vancomycin for patients with multiple Clostridium difficile recurrences. Clin Infect Dis 2013;56:309-10.

13. van Nood E, Vrieze A, Nieuwdorp M, et al. Duodenal infusion of donor feces for recurrent Clostridium difficile. N Engl J Med 2013;368:407-15.

14. Edlund C, Barkholt L, Olsson-Liljequist B, Nord CE. Effect of vancomycin on intestinal flora of patients who previously received antimicrobial therapy. Clin Infect Dis 1997;25:729-32.

15. Hamilton MJ, Weingarden AR, Sadowsky MJ, Khoruts A. Standardized frozen preparation for transplantation of fecal microbiota for recurrent Clostridium difficile infection. Am J Gastroenterol 2012;107:761-7.

16. Rohlke F, Stollman N. Fecal microbiota transplantation in relapsing Clostridium difficile infection. Therap Adv Gastroenterol 2012;5:403-20.

17. Silverman MS, Davis I, Pillai DR. Success of self-administered home fecal transplantation for chronic Clostridium difficile infection. Clin Gastroenterol Hepatol 2010;8:471-3.

18. Petrof EO, Gloor GB, Vanner SJ, et al. Stool substitute transplant therapy for the eradication of Clostridium difficile infection: 'RePOOPulating' the gut. Microbiome 2013;1:3-12.

19. Brandt LJ. American Journal of Gastroenterology Lecture: Intestinal microbiota and the role of fecal microbiota transplant (FMT) in treatment of C. difficile infection. Am J Gastroenterol 2013;108:177-85. 


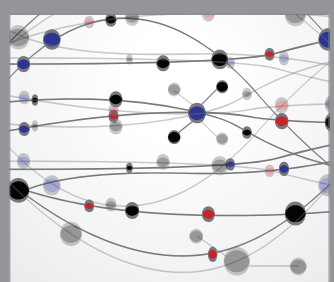

The Scientific World Journal
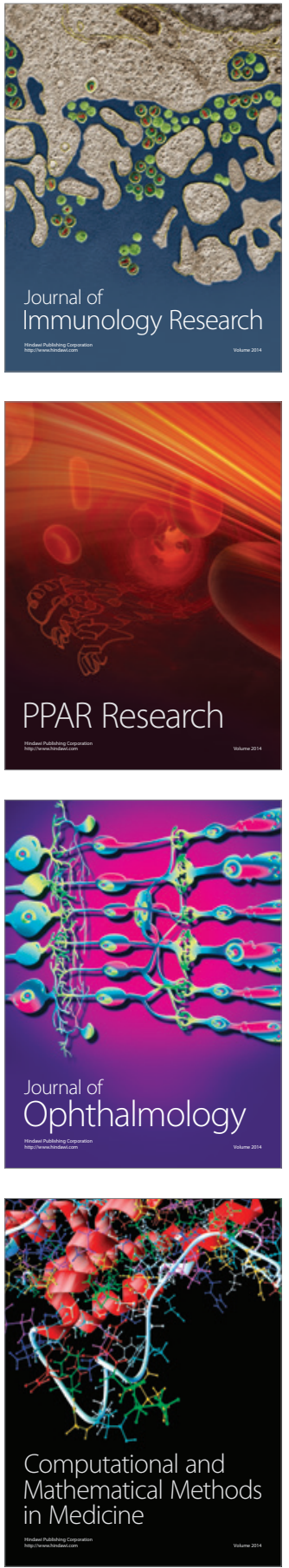

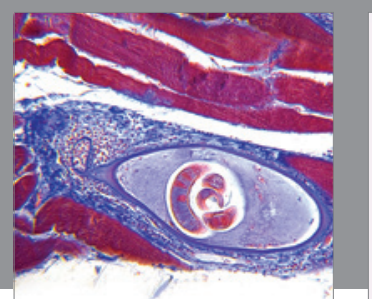

Gastroenterology Research and Practice

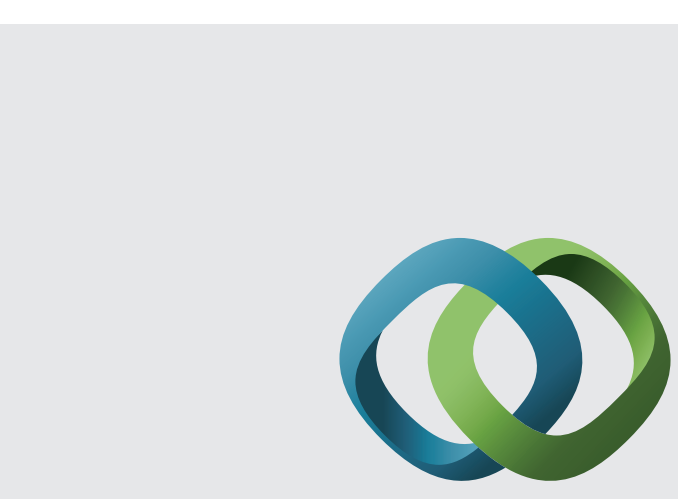

\section{Hindawi}

Submit your manuscripts at

http://www.hindawi.com
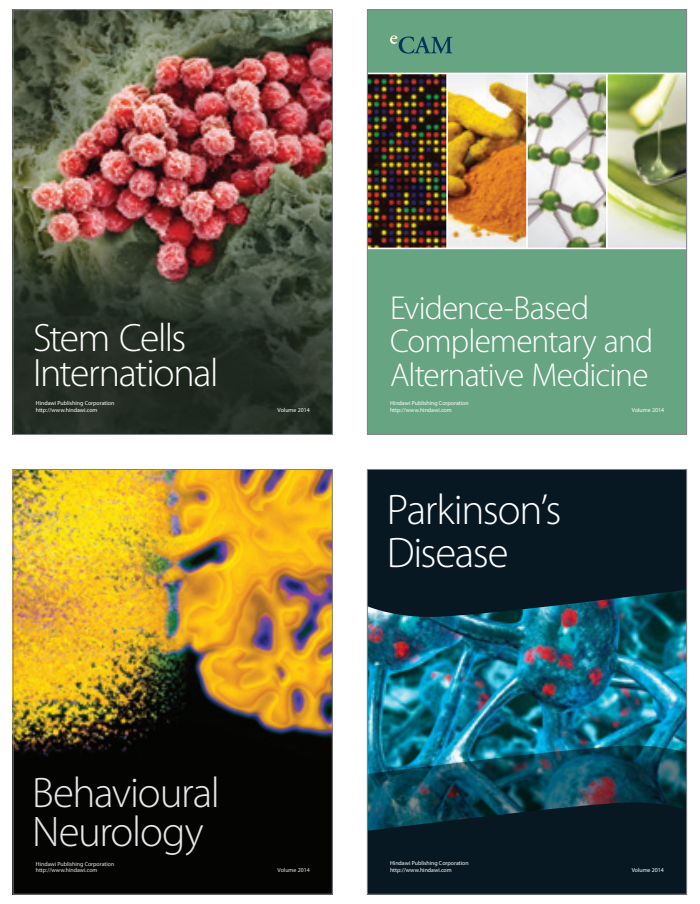
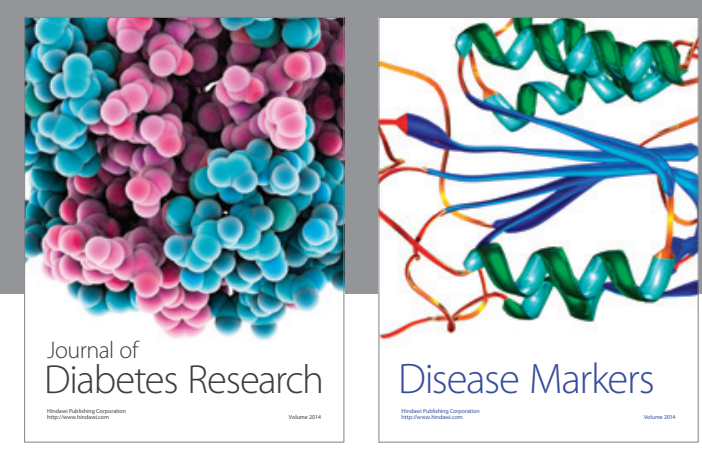

Disease Markers
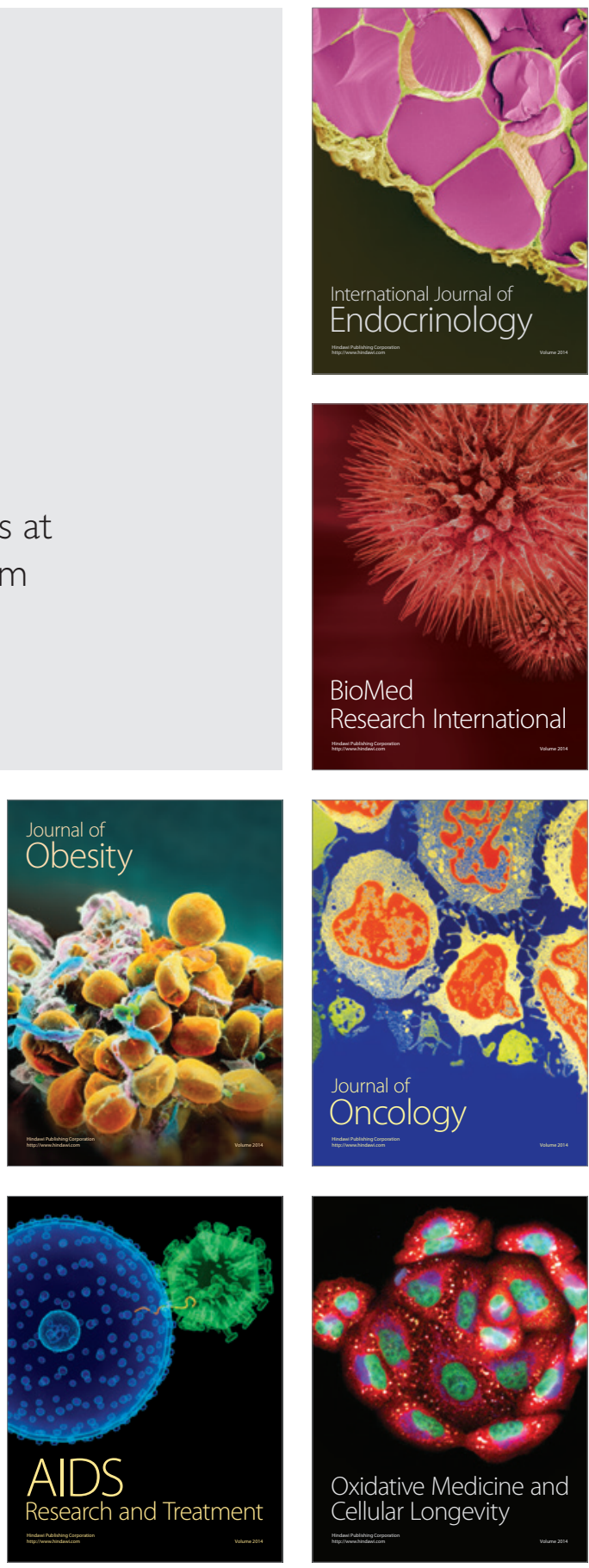